\title{
ALGUNOS ELEMENTOS PARA REFLEXIONAR ACERCA DEL QUEHACER PROFESIONAL Y LOS MODELOS CONVENCIONALES Y ALTERNATIVOS \\ DE DESARROLLO
}

Mary Hellen Burbano*

\begin{abstract}
RESUMEN
En los últimos tiempos se viene repensando a nivel de América Latina un proceso de Reconceptualización ${ }^{1}$ del Trabajo Social mas allá del llevado a cabo en los años 60, que marcó una profunda e importante huella dando apertura a la introducción de nuevos fundamentos para la formación y la acción, permitiendo un reposicionamiento de nuestra profesión en el marco de la realidad social. Hoy desde algunos discursos, el Trabajo Social se propone como una disciplina en tránsito hacia "un nuevo nivel de desarrollo del quehacer profesional", cuyo objetivo apunta hacia una apuesta para la construcción de espacios más abiertos proyectivamente hacia el futuro, para realizar mayores y mejores aportes, colaboraciones y proposiciones de parte de los profesionales".
\end{abstract}

Palabras claves: Desarrollo, trabajo social

\begin{abstract}
In the last times one comes rethink to level of Latin America a process from Reconceptualization of the Social Work beyond of the carried out one in the years 60, that a deep and important track marked giving opening to the introduction of new foundations for the formation and the action, allowing a re-positioning of our profession in the frame of the social reality. Today from some speeches, the Social Work sets out like a discipline in transit towards "a new level
\end{abstract}

\footnotetext{
" Trabajadora social de la Universidad del Valle. Estudiante de la especialización en Desarrollo comunitario de la misma Universidad. Coordinadora del programa de trabajo social de la Universidad del Valle-Sede Zarzal.

${ }^{1}$ Según Gustavo Parra es durante la reconceptualización que se instala la discusión y el análisis sobre el carácter científico de la profesión y que es en este proceso de búsqueda sobre el estatuto de la profesión, que se establece el debate en torno al método y su objeto. En síntesis, la referencia a la cientificidad del Trabajo Social, a la definición de su método y de su objeto nos remite a una discusión epistemológica sobre el Trabajo Social. Tomado de Articulo EL OBJETO Y EL TRABAJO SOCIAL. Algunas aproximaciones a la problemática del objeto en el Trabajo Social Publicaciones desde el Fondo.
} 
of development of the professional task", whose objective aims towards one bet for the construction of more opened spaces projectively towards the future, to make greater and better contributions, collaborations and proposals from the professionals".

Keywords: Development, social work

\section{INTRODUCCIÓN}

ए

$\mathrm{n}$ los últimos tiempos se viene repensando a nivel de América Latina un proceso de

Reconceptualización ${ }^{2}$ del Trabajo Social mas allá del llevado a cabo en los años 60, que

marcó una profunda e importante huella dando apertura a la introducción de nuevos fundamentos para la formación y la acción, permitiendo un reposicionamiento de nuestra profesión en el marco de la realidad social. Hoy desde algunos discursos, el Trabajo Social se propone como una disciplina en tránsito hacia "un nuevo nivel de desarrollo del quehacer profesional", cuyo objetivo apunta hacia una apuesta para la construcción de espacios más abiertos proyectivamente hacia el futuro, para realizar mayores y mejores aportes, colaboraciones y proposiciones de parte de los profesionales"3.

Por lo tanto, desde la disciplina se hace necesario:

El desarrollo de un práctica profesional contextualizada a los cambios sociales que emergen en el seno de la sociedad contemporánea y que necesariamente nos convocan a reprensar nuevas formas, nuevas estrategias, nuevos abordajes, nuevos objetos de estudio, nuevos sujetos de intervención e incluso nuevas intencionalidades y posiciones respecto al mundo circundante, a la realidad y a la construcción de futuros y presentes- que se adviertan como escenarios distintos a las condiciones de inequidad, exclusión y totalitarismo del actual modelo hegemónico (Delgado y Erazo, 2005:67)

En este sentido, la apuesta es hacia un Trabajo Social mas critico pero sobre todo mas propositivo, ese es el reto al que actualmente se invita a los profesionales no solo del Trabajo Social y a los especialistas en Desarrollo Comunitario, sino también a todos aquellos quienes

\footnotetext{
${ }^{2}$ Según Gustavo Parra es durante la reconceptualización que se instala la discusión y el análisis sobre el carácter científico de la profesión y que es en este proceso de búsqueda sobre el estatuto de la profesión, que se establece el debate en torno al método y su objeto. En síntesis, la referencia a la cientificidad del Trabajo Social, a la definición de su método y de su objeto nos remite a una discusión epistemológica sobre el Trabajo Social. Tomado de Articulo EL OBJETO Y EL TRABAJO SOCIAL. Algunas aproximaciones a la problemática del objeto en el Trabajo Social Publicaciones desde el Fondo.

${ }^{3}$ Tomado del www.uscr.com
} 
por decisión propia optan por trabajar en el campo del desarrollo humano ${ }^{4}$ constituido por realidades con altos grados de complejidad e incertidumbre, compuesto por escenarios donde se cruzan diversas dimensiones constitutivas de lo objetivo y subjetivo, de lo material e inmaterial.

Sin embargo, y a pesar de que algunas veces esta intencionalidad critico-propositiva se ubica de manera clara en el pensamiento por quienes optan por esta postura, resulta no siendo tan coherente en el discurso y a veces un tanto menos en la acción. Lo digo por muchas razones que asaltan mi preocupación. En estos días escuchaba a algunos estudiantes de pregrado de la Escuela, cuyo discurso mostraba un cierto criticismo radical, incluso paralizante que se instala en la lógica del movimiento pendular, tendencia en la que oscilan las corrientes hegemónicas y las contrahegemónicas desde las cuales ha sido concebido el mundo, entre otras el Capitalismo v.s Comunismo; que como bien la historia lo ha demostrado, no ofrecen una salida alternativa a las tensiones que hoy enfrenta el mundo y la sociedad. De la misma forma, la cotidianidad y la complejidad de la realidad nos ha demostrado que aunque la crítica es fundamental, esta no es suficiente.

En ese orden, la hipótesis de trabajo que no pretendo agotar en este ensayo, tiene que ver con la idea de que las orientaciones del quehacer profesional o mejor dicho de las acciones propuestas a partir de Trabajo Social, no solo a nivel general sino a partir de nuestra propia práctica, en el aquí y en el ahora desde escenarios locales e incluso microlocales, están directamente relacionados con la noción de desarrollo que alberga nuestro propio proyecto ético-político, hijo directo de los modelos convencionales o alternativos de desarrollo, según desde donde este sea concebido. Por lo tanto, es de interés del presente texto explorar con mas detenimiento las propuestas y algunos de los postulados en los que descansan los modelos convencionales y alternativos de desarrollo y desde allí analizar el sentido del quehacer profesional del Trabajo Social, como disciplina en transición hacia una postura más critica pero también más propositiva. Y desde aquí aportar algunos elementos para la reflexión frente a la propia acción de profesionales que desean aportar a la construcción de un mundo distinto, para el cual no existen recetas ni formulas mágicas.

Es así como, el presente ensayo se encuentra organizado en tres capítulos, el primero de ellos esta dedicado a revisar tangencialmente los modelos convencionales y la idea de desarrollo, en el segundo capitulo abordo aun más tangencialmente las propuestas del desarrollo alternativo desde la mirada de la antropología del desarrollo, disciplina que ha aportado a nuestra profesión y en general a todas las ciencias sociales, atreviéndome a pensar algunos elementos que he venido

\footnotetext{
${ }^{4}$ Entendido como un proceso que conduce a ampliar la gama de posibilidades que se ofrece a cada uno, Son en principio ilimitadas y pueden modificarse con el tiempo. Sin embargo cualesquiera que sea el estadio del desarrollo, supone que han de cumplirse tres condiciones esenciales: vivir largo tiempo y con buena salud; adquirir conocimientos y tener acceso a los recursos necesarios para disfrutar de un nivel de vida adecuado, sino se satisfacen estas necesidades, muchas otras posibilidades permanecerán inaccesibles. (Rist, citado en Carvajal: 2005)
} 
recogiendo en mi paso nuevamente por la academia, y en el tercer capitulo, en un intento de pensamiento critico-creativo y osado, intento exponer algunos elementos para pensar el quehacer profesional en contextos de desarrollo convencional pero con una franca mirada desde lo alternativo, para la construcción de un desarrollo y un futuro con rostro y sentido propio.

\section{MODELOS CONVENCIONALES Y DESARROLLO}

No es objeto de este ensayo revisar todos los modelos convencionales de desarrollo, aquí nos detendremos especialmente en el Neoliberal, que se ha caracterizado por la pretensión de imponer un pensamiento único intentando por todos los medios de convertir su propia concepción del mundo en verdad universal ${ }^{5}$ y de imponer una racionalidad económica dominada por la lógica del capital y del mercado.

Sin embargo, este modelo que prometió múltiples cambios para el mundo y un progreso homogéneo en términos económicos para la población mundial como lo proponen por ejemplo los modelos de polos de crecimiento, hoy está en crisis, ha incumplido sus promesas, pues no ha logrado dar respuesta a las preguntas que la sociedad plantea y tampoco brinda elementos para resolver problemas álgidos a los cuales nos enfrentamos, como aquellos que tienen que ver con la forma de relación entre la Población - naturaleza, el Estado y el capital, entre otros.

Es por esto que hoy viene siendo puesto en cuestión, ya que no cabe duda de que este modelo que pretende ser "universal" desde su implementación en los años 80s, ha venido ampliando las brechas de desigualdad y pobreza en el mundo, tal como se puede apreciar en las estadísticas de distribución mundial de INTERMON, por ejemplo, donde se muestra claramente como el $14.7 \%$ de la población ubicada en países del Norte consumen mas del $60 \%$ de recursos energéticos, minerales y alimentos, entre otros, y cómo en términos de desigualdad internacional se hallan datos como que el ingreso del 1\% mas rico de la población mundial equivale al del 57\% de la mas pobre (fuente: PNUD, 2002, citado en Carvajal, 2005).

Estas inmensas asimetrías propiciadas por el propio modelo neo-liberal apalancado en lógicas de modernización, competitividad, estado mínimo, desregularización y mercado, entre otras han contribuido a agravar problemas ambientales, sociales y políticos especialmente en países denominados del Sur (Estefanía, citado en Carvajal, 2005).

Actualmente, igual que hace mas de 50 años, luego de la segunda guerra mundial, los Estados Unidos han puesto en marcha un nuevo proceso de reestructuración de dominación donde se propone rediseñar el territorio del continente con un modelo de regulación a partir del

${ }^{5}$ La noción de hegemonía es definida por Ceceña como la capacidad de convertir la propia concepción del mundo en verdad universal, para lo cual se requiere establecer un orden Militar, Económico, Político y Cultural que se establece a través del consenso o la imposición. Diría Rist y a través del discurso. 
desarrollo de tecnología, el fortalecimiento de su fuerza militar, la militarización de las relaciones internacionales y la ocupación de territorios de forma simbólica y física a través de los procesos de globalización. Proceso de ampliación de la hegemonía que se da no solo en el campo de lo económico, sino también en lo político, lo cultural, en el lenguaje, etc. que amenaza la soberanía de las naciones, la democracia, la autonomía y libertad; pretendiendo tener el control total.

No obstante, la implementación de este modelo que cohabita con algunos rezagos del modelo Keynesiano y Cepalino para el caso de países latino americanos, ha contribuido a que países especialmente del sur, dependientes de las grandes economías del norte reconozcan la necesidad de revisar la carrera emprendida desde los años 80 s hacia los procesos de desarrollo como una meta absolutamente necesaria, entendida en términos de progreso económico a partir del aumento de la producción a como diera lugar. Y es precisamente en este contexto donde surgen preguntan ineludibles como: ¿Qué entendemos por desarrollo? ¿Qué tipo de desarrollo queremos? Y ¿a qué costo estamos dispuestos a obtenerlo?

Sin embargo, antes de pasar a abordar la naturaleza de algunos de estos interrogantes que atraviesa la vida de todos los seres humanos y más aún de los profesionales vinculados a proyectos y programas que agencian distintas nociones del "desarrollo", es importante detenernos en el análisis acerca del origen del discurso de este concepto tan utilizado a partir de los años 50s.

Acerca de la invención del concepto de desarrollo, varios autores coinciden en que su aparición se remonta al periodo histórico posterior a la segunda guerra mundial. En este sentido, Ceceña (1997), afirma que para este momento los Estados Unidos vio la necesidad de reformular su estrategia de reposicionamiento como hegemónica, recreando la relación de dominación con países de América Latina y el Caribe, con el objeto de proteger sus propios intereses, asegurar su soberanía, territorio y población, prevenir el surgimiento de cualquier tipo de expresión que amenazara su hegemonía, contar siempre con acceso a los mercados decisivos y a las fuentes de energía, recursos y materia prima, derrotar cualquier expresión en contra suya, garantizar libertad y seguridad, a partir de la ubicación de América Latina y El Caribe como una base de apoyo para la competitividad de EEUU; convirtiéndolos en mercados cautivos y estrechando mas las relaciones de intervención y dependencia.

En esta misma dirección, Rist (2002) afirma que es precisamente en este periodo histórico donde tiene lugar el refinamiento de la política exterior de los Estados Unidos orientada a lograr la estabilidad económica y la contención del avance comunista en el mundo y, añadiría Ceceña (1997), a reposicionarse como Hegemónico ante las posibles amenazas, a través de 4 estrategias centrales, expuestas por el presidente Truman en uno de los discursos proclamados en 1948 que después daría origen a la aparición de la Doctrina Truman ${ }^{6}$.

\footnotetext{
${ }^{6}$ En un discurso al Congreso, el presidente de Estados Unidos Harry S. Truman anunció la puesta en marcha de una política exterior orientada a lograr la estabilidad económica y la contención del avance comunista en el mundo. La Doctrina Truman intensificó la lucha ideológica iniciada entre el Este y el
} 
Estos lineamientos estarían centrados en 4 postulados: 1 Continuar apoyando la naciente ONU antes SDN, ahora en New York antes en Ginebra, 2. La creación de la OTAN como una organización para la defensa común ante la posible amenaza de la Unión Soviética, 3. La contribución para la reconstrucción de Europa devastada después de la guerra a través del Plan Marshall, y por último, el punto que según este autor, es en el que se apalanca el discurso del desarrollo e inauguraría su era, dado a partir de la formulación de una nueva forma de relaciones internacionales basada en la extensión de los beneficios de los adelantos tecnológicos a todos los países de América Latina, con el fin de promover el mejoramiento de estas regiones señaladas como económicamente atrasadas o "subdesarrolladas" que podrían poner en riesgo su propia prosperidad. Aunque esta no fue la primera vez en que se usara este término, pues ya lo habían hecho clásicos de la economía y la política desde ese momento y hasta ahora:

Los distintos usos de la palabra tienen en común que presentan al "desarrollo" conforme a la tradición occidental del concepto, como un fenómeno intransitivo que, simplemente, "se produce" sin que se pueda cambiar nada de el.

La aparición del "subdesarrollo" sugiere no solamente la idea de un cambio posible dirigido hacia un estado final, sino sobre todo la posibilidad de provocar ese cambio. No se trata solamente de tomar nota de que las cosas "se desarrollan", sino de que se podrán desarrollar. El "desarrollo" toma entonces un estado transitivo (el de una acción ejercida por un agente distinto a él) correspondiendo a un principio de organización social, mientras que el "subdesarrollo" será considerado como un estado que existe "naturalmente" es decir sin causa aparente" (Rist: 2002).

La implementación de este discurso aparece de la mano con varios cambios políticos y de pensamiento que intentan reemplazar la antigua relación colonizadores/colonizados, donde cada uno respondía a mundos distintos puestos en un orden de jerarquía, por una relación en la que todos los Estados serían iguales en el plano de los derechos, en el que se promovería la descolonización y se fundaría un nuevo escenario en el que fuese posible pasar de obrero a capitalista, de pobre a rico, etc. a fuerza de perseverancia y trabajo y en el que se prueba la necesidad de la intervención externa a partir de la justificación moral de no de ver caer en manos de la miseria a países "subdesarrollados" por razones desconocidas, mientras otros gozan de la abundancia que día a día se reproduce y no dejarían de crecer; pero también por razones prácticas donde todos como resultado de un esfuerzo colectivo, ayudarían a los otros a alcanzar la prosperidad a partir de la ampliación de la producción y de un uso eficiente de recursos naturales y humanos, incluso poniendo estos recursos a disposición de los otros.

Oeste al final de la II Guerra Mundial, sobre todo en Europa, y dio paso a un periodo de conflicto social y político a escala mundial conocido como Guerra fría. Biblioteca de Consulta Microsoft ${ }^{\circledR}$ Encarta ${ }^{\circledR}$ 2005. (C) 1993-2004 Microsoft Corporation. Reservados todos los derechos. 
Así, el desarrollo aparece desde el discurso como la posibilidad para la población de acceder al tan anhelado progreso en términos especialmente económicos y materiales, que se promueve desde lo cotidiano, los discursos gubernamentales y privados y a través de los medios de comunicación; en lo práctico el desarrollo aparece como el resultado de la implementación de un modelo que privilegia la economía, se desliga de lo social y hace de la política un instrumento de intervención que permita crear las condiciones para su reproducción ampliada, aumentando las brechas de pobreza y desigualdad económica y social; y en lo político, se devela como una estrategia simbólica para el ejercicio del poder y la dominación, ya que no es desconocido que para que puedan existir ricos deben existir los pobres.

En conclusión, la concepción de desarrollo agenciada por el modelo convencional, que no es definido por la economía sino por las personas que la piensan y construyen, es decir generalmente por las clases dominantes que son quienes detentan mayoritariamente el poder económico, el poder político y ahora científico y tecnológico, es concebido como un concepto a priori único, universal y objetivo, por lo tanto, no toma en cuenta la dimensión de lo humano, la cultura ${ }^{7}$, el territorio, la historia, el contexto ni la espacio-temporalidad de los procesos sociales, entre otros elementos. Este tipo de desarrollo esta dado en términos puramente económicos, como fin en si mismo, único motivo de base para la felicidad, la calidad de vida, el bienestar y el progreso que se debe alcanzar, no importando a través de que medios o a que precio. Es una noción de desarrollo homogénea y totalizante.

Por ejemplo, para Escobar(1994), desde este discurso denominado Liberal, nacen propuestas que aunque argumentan promover un desarrollo sostenible, son tan solo estrategias discursivas que permite hacer más eficiente y maximizar el uso de los recursos naturales que intervienen en los procesos de producción capitalista para la generación de más productos, con "menos daño al medio ambiente".

Según la lógica de este discurso, la naturaleza más que tener un valor en sí mismo como fuente de vida, tiene un valor monetario que lo convierte en objeto de privatización a manos de los seres humanos; esta propuesta lo único que permite es que el capital continúe su reproducción sin ser puesto en cuestión y sin promover que los sujetos tomen conciencia de que el problema ambiental se deriva de la forma como el hombre se esta relacionando con la naturaleza, es decir de que es un producto directo del desarrollo del actual modelo económico. Es así como, la propuesta liberal tiene el objetivo claro de preservar los recursos naturales para permitir que la cultura económica siga siendo percibida como natural y dada de una vez por todas, cuya función es la de regular las relaciones sociales por medio de sus leyes y continuar perpetuando un determinado orden internacional propicio para la reproducción del capital y los centros de poder.

\footnotetext{
${ }^{7}$ Entendida como un esquema históricamente trasmitido de significaciones representadas en símbolos, concepciones por medio de las cuales la gente se comunica, perpetúan y desarrollan su conocimiento y actitud frente a la vida.
} 
En este escenario el trabajo social, tiene muy poco alcance y no logra corresponder a los retos que imponen los nuevos tiempos, tal como lo afirma Parra (2005):

En cuanto aporte a la discusión, consideramos que los esfuerzos por elaborar definir un objeto en el Trabajo Social, desde la perspectiva hegemónica en el campo de la epistemología positivista, nos conduce a un callejón sin salida como profesión, sea porque nos reduce a una mera técnica, sea porque limita las posibilidades de intervención profesional. Creemos que debemos superar los meros análisis epistemológicos, entendiendo que las categorías además de tener un componente lógico tienen un componente histórico, por lo cual son esencialmente ontológicas. Desde allí se abren posibilidades para el análisis, la reflexión, la profundización de nuestra intervención y desarrollo profesional. Desde allí la práctica profesional requiere, este desafío de lectura de la realidad, un análisis que permita superar visiones rutinarias y estáticas, que permita poner en juego la creatividad, las competencias profesionales, las funciones profesionales.

En este sentido, y teniendo en cuenta que ya no somos presa de ese pretendido objetivismo y que por el contrario estamos cargados de simbolismos y significados, que nuestra historia de vida va con nosotros a todas partes, que es imposible despojarse de los valores y concepciones del mundo antes de ejercer el rol profesional, se hace necesario hacer conciencia de que la mirada hacia el otro, la propia concepción de desarrollo, en vez de ampliar democracia y reconocer los otros y las otras en la diferencia, se puede convertir en un vehiculo para la reproducción inconsciente de esquemas de exclusión, de prejuicios raciales y de clase frente la población participante.

De igual manera, y en esta misma línea es necesario reconocer que toda acción lleva implícita una apuesta hacia un determinado orden social, una determinada apuesta de desarrollo y que si bien todos llevamos consigo un proyecto ético político, la mejor apuesta es hacia el reconocimiento de la ética como un ejercicio constante de reflexión que logre ubicar a los sujetos como centro de las decisiones, promoviendo el ejercicio de la libertad, del pluralismo, y sobretodo del respeto por la diferencia para la construcción de consensos acerca de lo que queremos y esperamos de nuestro futuro como sujetos, grupos, organizaciones, comunidades y sociedades y no la imposición de una determinada concepción de desarrollo, sujeto y sociedad, cayendo en la negación del otro y la imposición de la cual hemos sido presa desde hace muchos siglos.

Por lo tanto, la invitación es no solo a descubrir y a reconocer la diversidad de discursos, si no a repensar la lógica de las prácticas para definir a qué proyecto de sociedad le estamos apostando, poniendo en cuestión los modelos de desarrollo, no solo para elaborar elementos para la acción, sino para aportar a la construcción de un modelo de desarrollo como el resultado de un proceso de redescubrimiento raizal, compartido, democrático y coherente. 


\section{MODELOS ALTERNATIVOS DE DESARROLLO}

Mas allá de la lógica que proponen las entradas alternativas como el etnodesarrollo, el desarrollo local, el desarrollo endógeno y el desarrollo sostenible, en los cuales según Carvajal(2005) hay un rescate de la concepción del desarrollo para sus actores, de la autonomía para determinar como quieren ser felices, dándose una revalorización de lo propio potenciando los recursos locales, el desarrollo es concebido como un concepto diverso, complejo e inacabado, como proceso y producto socio-histórico, cuyo sentido es susceptible de trasformar y resignificar y en el que confluyen distintos tipos de fuerzas, tensiones y conflictos que median para definir el futuro.

En este orden de ideas, hoy considero necesario revisar algunos de los elementos que permiten pensar un desarrollo de tipo alternativo en términos de proceso, que si bien ofrece un lugar vital a la localidad como punto de referencia, trasciende y se relaciona de manera reciproca e integral con el ámbito de lo global, además de ubicar como lineamiento central la participación de la población local en la definición orientación y manejo de su propio desarrollo, alcanzando allí la participación su máximo nivel: el de la decisión y gestión donde la gente deja de ser un objeto para convertirse en sujeto.

Por lo tanto, la noción de Desarrollo que aquí se esboza es asumida como un proceso de construcción social e histórica, que es dotado de contenido a partir de las características propias de quien la defina de manera prescriptiva y descriptivamente, no obstante, conjuga necesariamente la presencia de condiciones materiales e inmateriales que hacen parte de todas las dimensiones de la vida de los sujetos desde lo cotidiano y colectivo (sujeto-sujeto) hasta su participación en lo público (sujeto-Estado). Esta noción, esta definida en términos de proceso en continuo cambio, permitiendo ser coherente con lo que se espera del futuro. Obviamente en el ejercicio se retoman algunos aspectos reivindicados por modelos Convencionales y otros denominados alternativos.

La idea fuerza que subyace a este planteamiento es que debe existir un equilibrio entre lo material y lo inmaterial. Debe ser un desarrollo con "rostro humano, centrado en lo subjetivo, pero sin perder de vista que es necesario que la población cuente con condiciones dignas de vida, reconocidas por un Estado de carácter democrático que le apueste no solo a la construcción real de consensos, al reconocimiento de la pluralidad y al respeto por la diferencia, donde quienes manden lo hagan obedeciendo realmente la voluntad de los actores.

Para terminar este capitulo, me atrevo a presentar algunos elementos que contribuirían a pensar un modelo alternativo que hiciese posible el desarrollo como producto de la construcción social y que permita repensar el piso desde el cual nos estamos parando como profesionales para intervenir en procesos de desarrollo: 
- Estado que controle el mercado y regule las relaciones sociales pero que de espacio a la participación de la ciudadanía.

- Que proponga la región como escenario de enclave entre lo local y lo nacional definido a partir de las dinámicas de los procesos sociales.

- Énfasis en la economía local, regional y nacional.

- Que no se mueva en lógicas de dependencia y no establezca relaciones de intercambio en términos desiguales.

- Estado que genere condiciones de igualdad material para la población, condiciones dignas de vida.

- Promueva los valores de lo colectivo, la solidaridad, el bien común y privilegie a los seres humanos ante los objetos materiales.

- Integración de cambio social y crecimiento económico fortaleciendo economía interna

- Relación de equilibrio con la naturaleza; donde la naturaleza no sea tratada como mercancía sino que se reconozca su valor intrínseco de vida.

- Un Estado profundamente Democrático: que privilegie el consenso, el pluralismo y reconozca y respete la diferencia

- Que promueva un tipo de desarrollo raizal y autónomo

- Estado con el poder del monopolio de las armas

- Mayor control del legislativo sobre el ejecutivo

- Un Estado transparente que permita un verdadero ejercicio del control social

- Protección del mercado interno restringiendo importaciones de productos producidos en el país.

- Que privilegie el dialogo, el consenso y los acuerdos sobre la salida violenta

- Que promueva canales efectivos de participación de la población y las comunidades locales en la toma de decisiones (económicas y políticas)

- Estado descentralizado que otorgue poder de decisión y recursos a entes locales

- Definición de Políticas Públicas complementarias para potenciación de calidad de vida de la población y no focalizadas y asistencialistas

- Que promueva Programas y proyectos coherentes con la especificidad cultural de las comunidades (respetando y reconociendo formas autónomas de ser)

- Que promueva el fortalecimiento de la cultura democrática, ciudadana y política.

- Énfasis en lo local y Equilibrio entre lo local y lo global

- Manejo de poder legitimo por parte del Estado.

- Defensa de los derechos de las minorías

- Un Estado garante de los derechos humanos

- Fortalecimiento de la relación entre elegido y electores (mayor control y no entrega total del poder)

- Establecimiento de relaciones estrechas entre las economías de América del Sur

- Que reconozca las diferencias entre hombre y mujeres y sus condiciones para participar

- Entre otros... 


\section{TRABAJO SOCIAL Y DESARROLLO ALTERNATIVO}

Desde el ámbito de la investigación y la acción profesional desde el trabajo social, considero importante traer a colación lo que algunos colegas que se mueven desde la corriente criticopropositiva vienen proponiendo; ellos postulan que es posible el acercamiento de los profesionales a los fenómenos sociales para interpretarlos y comprenderlos e incluso intervenirlos como formas y vividas por los sujetos quienes la construyen y atribuyen sentido, desde tres posturas básicas que considero estan directamente relacionadas con la concepción desde el cual se pare uno para intervenir el desarrollo.

La primera postura es hija directa del legado del positivismo:

\footnotetext{
Que como corriente de pensamiento se basa en el supuesto carácter de neutralidad que debe asumir la ciencia y las diferentes profesiones que hacen ciencia. La realidad social que se estudia se puede describir y explicar, se pueden identificar las "leyes" que predicen supuestos comportamientos sociales, pero bajo ninguna circunstancia se puede tomar partido para cambiar esa sociedad. La sociedad es inmutable, inamovible, no se pueden esperar cambios de ninguna naturaleza, menos inducirlos. (Marcos Chinchilla,2006)
}

Es decir desde esta posición la acción profesional esta destinada a la reificación de un status quo dado, inamovible y definido de una vez por todas, esta postura implicaría moverse aún en uno de los modelos convencionales, el modelo dominante que ve a los procesos sociales como objeto de estudio y de intervención para su descripción e interpretación entre otras, o puede significar también estar del lado del criticismo a ultranza que paraliza, porque si bien la realidad se puede cambiar serían unos pocos los llamados a hacerlo, los antiguos proletarios que el sistema se ha encargado de ir diezmando poco a poco, cuya condición hoy es considerada por muchos como un lujo, para quienes no logran y no lograrán por lo menos en el futuro mas cercano, gozar de condiciones de trabajo digno a causa del cambio en las políticas laborales que cada vez mas violenta y deconstruye las condiciones de autosuficiencia y autonomía de países del Sur.

Una segunda opción tendría que ver en el campo de la investigación especialmente, con la producción de conocimiento socialmente relevante, a partir de la generación de conocimiento colectivo que permita la comprensión no solo del contexto político en el que se desarrolla la intervención social, sino también por la posibilidad de que este conocimiento pueda ser usado para fortalecer las formas organizativas ya existentes que participan en los procesos de construcción de conocimiento u organización colectiva por ejemplo, permitiendo fortalecer las acciones que estos sectores sociales involucrados piensan o vienen desarrollando (Chinchilla, 2005).

Se podría pensar, que esta articulación está dirigida no solo hacia el desarrollo de proyectos de investigación cuyos resultados aporten al fortalecimiento de las formas organizativas existentes 
y su accionar, sino también hacia la participación del profesional en la orientación de los propios procesos sociales a través del acompañamiento por ejemplo de procesos de identificación de sentidos socialmente compartidos, que facilitan la negociación y concertación colectiva y autónoma por parte de los sujetos involucrados, quienes son los encargados en últimas de dar forma a los proyectos y de tomar sus propias decisiones de acuerdo a sus intereses, expectativas y visiones del mundo. Allí los sujetos de intervención se sitúan en el centro de las decisiones, promoviendo quizás procesos de construcción de sujetos sociales y políticos.

Aquí el papel del trabajador social se situaría dentro del campo de la asesoría, acompañamiento y retroalimentación de los procesos de construcción colectiva, sin que necesariamente haya un nivel de adhesión mayor por parte del profesional. Esta opción es la que generalmente asumimos dentro de nuestro ejercicio profesional, donde se intenta promover el fortalecimiento de los sectores u organizaciones populares a través de la participación directa, que se presenta como una alternativa de construcción y ensayo de poder desde la base social en contextos de lo local.

Sin embargo, existe una tercera opción que viene siendo ventilada quizás desde el propio movimiento de reconceptualización del trabajo social y que hoy retoma fuerza desde los enfoques de la acción crítica y se convierte en un reto que confronta nuestro propio proyecto de vida profesional y político. Esta opción, es una elección que implica adoptar una posición mas activa a través del desarrollo de un ejercicio profesional donde los procesos sociales dejan de ser vistos como una simple oportunidad para acercarse a actores colectivos jalonadores de proyectos alternativos o no de desarrollo y de reconfiguración social, esta elección implica hacer parte integral de el, poniendo en juego el propio proyecto profesional concebido no como algo aislado, sino necesariamente inspirado en y articulado a proyectos societarios (Montaño, 2005).

Esto supone poner en juego el proyecto ético-político de cada profesional y ser concientes de la importancia de que nuestra acción profesional se desarrolle más allá de los marcos institucionales desde donde tradicionalmente surge, partiendo de realidades concretas como un eslabón de enclave que permita la integración entre el saber académico y popular, participando de la construcción de redes sociales de apoyo entre distintos y amplios sectores que se expresan en contra de desigualdades, injusticias sociales o realidades insatisfactorias y que promueven el ejercicio de un mayor nivel de control y poder de decisión en la definición de proyectos colectivos, donde sus actores se asumen menos como objetos y mas como sujetos constructores de realidades.

En este contexto la acción profesional se vincula de manera directa con una estrategia que surge desde un determinado sector y como respuesta deliberada a una situación de insatisfacción. Aquí el profesional puede hacer uso de su saber político como una estrategia de acción para la promoción de una auténtica participación social en la exigencia del respeto por los derechos humanos, la democracia y la construcción de ciudadanía (Chinchilla, 2005).

Y es claro que como profesionales del área social no podemos ser ajenos a los hechos de la vida política, económica y organizativa de nuestro país, ni a la aparición de nuevos actores 
colectivos que surgen a partir de las necesidades y problemas propios del modelo económico y político imperante, si pretendemos aportar al desarrollo de la sociedad en términos integrales.

Esta opción permite que el trabajador social se articule de una manera más orgánica y activa en la construcción de espacios de participación, empoderamiento y desarrollo humanizado que permitan un mejor posicionamiento de amplios sectores de la población ante una realidad globalizada y globalizante construyendo con otros un intento transitorio de noción de desarrollo que guié no solo la acción.

\section{BIBLIOGRAFÍA}

BETANCOURT, Lady J y BURBANO, Mary H (2002): Diagnóstico de Familia con Participación comunitaria, Municipio de Caloto. Escuela de Trabajo Social y Desarrollo Humano, Universidad del Valle, Santiago de Cali.

CARVAJAL, Arizaldo (2005). Desarrollo y Cultura. Elementos para la reflexión y la acción. Escuela de Trabajo Social y Desarrollo Humano. Universidad del Valle. Santiago de Cali.

CARVAJAL, Arizaldo y otros (2005). "Desarrollo, identidad y acciones colectivas", en: Sujetos sociales, acciones colectivas y Trabajo Social. Seminario permanente. Escuela de Trabajo Social y Desarrollo Humano. Universidad del Valle. Santiago de Cali.

CARBALLEDA, Alfredo Juan Manuel (2000). La Reconceptualización Hoy Universidad de Buenos Aires. Investigador. Publicación virtual. Revista Margen

CECEÑA, Ana Esther (2003) América Latina en la Geopolítica el poder. Alternativa sur. Bogotá.

CHINCHILLA, Marcos (2005). Acción colectiva e intervención profesional del Trabajo Social: limites y posibilidades para la construcción de ciudadanía. Ponencia presentada en el I encuentro latinoamericano de Trabajo Social Crítico. Santa Fe de Bogotá. Colombia.

DELGADO Alexandra y ERAZO, David (2005)." Identidad colectiva: proceso y producto social "en: Sujetos sociales, acciones colectivas y Trabajo Social. Seminario permanente. Escuela de Trabajo Social y Desarrollo Humano. Universidad del Valle. Santiago de Cali.

DE SOUZA, Boaventura (2003): Globalización y Democracia. Ponencia presentada al Foro Mundial Social Temático, Cartagena de indias.

ESCOBAR, Arturo (2001): "Política Cultural y Cultura Política. Una Nueva mirada sobre los movimientos sociales", en: trasformaciones políticas y cambio cultural. Editorial Trotta, Madrid.

ESCOBAR, Arturo (1994). "El desarrollo Sostenible: dialogo de discursos", En: Integración y equidad, Corporación SOS. Viva la ciudadanía. Santa Fe de Bogotá.

MONTAÑ, Carlos (2005): Un proyecto ético-político para el Trabajo Social. Ponencia presentada en el I encuentro latinoamericano de Trabajo Social Crítico. Santa Fe de Bogotá. Colombia.

RIST, Gilbert (2002). El desarrollo: historia de una creencia occidental. Universidad Complutense de Madrid. La Catarata, Madrid.

PARRA, Gustavo (2005). EL OBJETO Y EL TRABAJO SOCIAL

Algunas aproximaciones a la problemática del objeto en el Trabajo Social. Publicación virtual desde el fondo.

Pagina Web. www.uscr.com

Recibido: Junio 30 de 2006

Aprobado: Septiembre 18 de 2006

ALGUNOS ELEMENTOS PARA REFLEXIONAR ACERCA DEL QUEHACER...-MARY HELLEN BURBANO 